A population-based study. Allergol Immunopathol (Madr). 2016;44(6):563-570. doi: 10.1016/j.aller.2016.03.003.

3. Coon ER, Quinonez RA, Moyer VA, Schroeder AR. Overdiagnosis: How Our Compulsion for Diagnosis May Be Harming Children. Pediatrics. 2014;134(5):1013-23. doi: 10.1542/peds.2014-1778.

4. Burks AW, Tang M, Sicherer S, Muraro A, Eigenmann PA, Ebisawa M, et al. ICON: Food allergy. J Allergy Clin Immunol. 2012;129(4):906-20. doi: 10.1016/j.jaci.2012.02.001.

5. Iorio RA, Del Duca S, Calamelli E, Pula C, Lodolini M, Scamardella F, et al. Citrus Allergy from Pollen to Clinical Symptoms. PLoS One. 2013;8(1):e53680. doi: 10.1371/ journal.pone.0053680.

6. Sole D, Jacob CMA, Pastorino AC, Porto Neto A, Burns DA, Sarinho ESC, et al. O conhecimento de pediatras sobre alergia alimentar: estudo piloto. Rev Paul Pediatr. 2007; 25:311-6.

Correspondencia: Daniel Enrique Mendoza Quispe

Dirección: Parque Unión Panamericana 136. Lima, Perú

Teléfono: +51987829720

Correo electrónico: daniel91emq@gmail.com

\section{PERCEPCIÓN SOBRE PROMOCIÓN FARMACÉUTICA Y PRESCRIPCIÓN DE MEDICAMENTOS EN MÉDICOS DE LIMA, PERÚ 2016}

\author{
PERCEPTIONS ON PHARMACEUTICAL \\ PROMOTION AND MEDICINE PRESCRIPTION \\ OF PHYSICIANS IN LIMA, PERU 2016
}

\author{
Rafael O. Florián-Castro. 1,a.
}

Sr. Editor. En los últimos 50 años, los visitadores médicos son el principal canal de comunicación para transmitir información sobre mercadotecnia de productos farmacéuticos mediante la visita a médicos. Esta actividad representa el $60 \%$ de los gastos de ventas de la industria farmacéutica. En Estados Unidos la información brindada en la mercadotecnia de los visitadores médicos está regulada y supervisada para asegurar su consistencia con lo estipulado por la Agencia de Administración de

\footnotetext{
Escuela de Posgrado. Universidad San Ignacio de Loyola. Lima, Perú. Ingeniero, Magister y Doctor en Administración.

Recibido: 07/06/2018 Aprobado: 05/09/2018 En línea: 21/12/2018
}

Los resultados son parte de la tesis doctoral de Florián Castro R. (2017). Relación entre promoción farmacéutica y prescripción de medicamentos en Perú [Tesis doctoral]. Lima: Universidad San Ignacio de Loyola. Escuela de Postgrado 2017. Citar como: Florián-Castro RO. Percepción sobre promoción farmacéutica y prescripción de medicamentos en médicos de Lima, Perú 2016. Rev Peru Med Exp Salud Publica. 2018;35(4):710-1.doi:10.17843/rpmesp.2018.354.3718.
Alimentos y Medicamentos (FDA). En el 2010, el $11 \%$ de los médicos estadounidenses tenían restringido el contacto con visitadores médicos y $34 \%$ tenían algunas restricciones para contactar con los visitadores médicos ${ }^{(1)}$.

Los medicamentos son promocionados por los visitadores médicos mediante la exposición de sus principales características y bondades, o la entrega de muestras médicas para persuadir a médicos de prescribirlos ${ }^{(2)}$; dichas actividades requieren que los visitadores médicos tengan formación y adiestramiento adecuados, además de integridad ética para presentar información sobre medicamentos y realizar otras actividades de promoción de sus productos de manera correcta y responsable ${ }^{(3)}$.

Los criterios éticos para la promoción de medicamentos constituyen un estándar para apoyar y fomentar una mejor atención de la salud mediante el uso racional de medicamentos. La promoción a cargo de los visitadores médicosdebeconsiderarunusoadecuadodemedicamentos, mediante una presentación objetiva de sus propiedades, sin exagerar sus características. En Yemen un estudio demostró que las relaciones entre médicos y la industria farmacéutica generan conflictos con los criterios éticos de promoción de medicamentos. Los médicos contribuyen a establecer actividades promocionales indeseables cuando solicitan diversos beneficios, a los que consideran tienen derecho, a cambio de prescribir medicamentos. Por otro lado, los bajos ingresos de los médicos contribuyen a que la industria farmacéutica ofrezca promociones y beneficios, como ofertas de viajes, alojamiento en hoteles y expectativas de algunas donaciones de fondos ${ }^{(4)}$. Es importante destacar que la situación en el Perú no es muy diferente, por las bajas escalas remunerativas.

Como parte de la tesis doctoral del autor, se realizó un estudio transversal mediante la utilización de un cuestionario de 24 preguntas para determinar la relación entre la promoción farmacéutica y la prescripción de medicamentos. El cuestionario incluyo un párrafo introductorio que indicaba al participante el carácter voluntario, la confidencialidad en el manejo de los datos recopilados y su consentimiento en usar estos para el estudio. El diseño del cuestionario se basó en la revisión de literatura científica, la validación de cinco expertos (midiéndose suficiencia, claridad, coherencia y relevancia con la $\mathrm{V}$ de Aiken) y aplicándose una prueba piloto a 24 médicos.

Queremos describir la respuesta de los médicos respecto a dos preguntas ¿Existe conflicto de tipoético entre la promoción farmacéutica y los médicos al prescribir medicamentos? y ¿La promoción farmacéutica (visita médica, muestras médicas, etc.) está debidamente regulada en el Perú por el MINSA y DIGEMID? La encuesta se realizó entre enero y abril del 2016 a 2731 médicos de alto nivel prescriptor (buena cantidad de recetas captadas por la auditoria CloseUp International) y que laboran en Lima Metropolitana. Los médicos participantes laboraban en centros de salud del MINSA, Essalud, clínicas 
Tabla 1. Percepción sobre promoción farmacéutica y prescripción de medicamentos en médicos de Lima, Perú 2016

\begin{tabular}{|c|c|c|c|c|c|c|}
\hline & \multicolumn{2}{|c|}{ Mujeres } & \multicolumn{2}{|c|}{ Hombres } & \multicolumn{2}{|c|}{ Total } \\
\hline & $\mathbf{n}$ & $\%$ & $\mathbf{n}$ & $\%$ & $\mathbf{N}$ & $\%$ \\
\hline \multicolumn{7}{|c|}{$\begin{array}{l}\text { ¿Existe conflicto de tipo ético entre la promoción farmacéutica y } \\
\text { los médicos al prescribir medicamentos? }\end{array}$} \\
\hline Ningún conflicto & 86 & 47,3 & 132 & 43,3 & 218 & 44,8 \\
\hline Algún conflicto & 82 & 45,1 & 129 & 42,3 & 211 & 43,3 \\
\hline Mucho conflicto & 12 & 6,6 & 35 & 11,5 & 47 & 9,7 \\
\hline Conflicto total & 2 & 1,1 & 9 & 3,0 & 11 & 2,3 \\
\hline \multicolumn{7}{|c|}{$\begin{array}{l}\text { ¿La promoción farmacéutica (visita médica, muestras médicas, } \\
\text { etc.) está debidamente regulada en el Perú por el MINSA y } \\
\text { DIGEMID? }\end{array}$} \\
\hline Nada de acuerdo & 16 & 8,8 & 37 & 12,1 & 53 & 10,9 \\
\hline Poco de acuerdo & 80 & 44,0 & 139 & 45,6 & 219 & 45,0 \\
\hline De acuerdo & 70 & 38,5 & 110 & 36,1 & 180 & 37,0 \\
\hline Muy de acuerdo & 16 & 8,8 & 19 & 6,2 & 35 & 7,2 \\
\hline
\end{tabular}

MINSA: Ministerio de Salud, DIGEMID: Dirección General de Medicamentos, Insumos y Drogas

privadas y consultorios particulares. Se consideraron médicos generales, ginecólogos, pediatras, oftalmólogos e internistas. 487 médicos respondieron la encuesta (363 en la modalidad virtual y 124 de manera presencial).

El 44,8 \% de los médicos consideró que no existía ningún conflicto ético entre la promoción realizada por la industria farmacéutica y la prescripción de dichos medicamentos $(47,3 \%$ en mujeres y $43,3 \%$ en varones). Por otro lado, $55,9 \%$ de médicos esta poco o nada de acuerdo con el enunciado de que la promoción farmacéutica está debidamente regulada en el Perú (47,3\% mujeres y $42,3 \%$ hombres) (Tabla 1).

El Ministerio de Salud publicó el 14 de junio de 2017 el proyecto de norma técnica que establece criterios éticos para la promoción y publicidad de productos farmacéuticos y productos sanitarios ${ }^{(5)}$. Algunos aspectos destacados de la propuesta incluyen que las farmacéuticas son responsables de las declaraciones, informaciones y todas las actividades que realicen los visitadores médicos en los establecimientos de salud, y que estos mismos deberán comunicar a sus representadas cualquier información que reciban de los médicos relativa al uso de productos farmacéuticos y las reacciones adversas con la finalidad de contribuir con la farmacovigilancia. La propuesta también establece no condicionar la prescripción mediante cursos o congresos y prohíbe el ofrecimiento de incentivos.

Se evidencia la necesidad de transparentar la relación entre los médicos y otros profesionales de salud con la industria farmacéutica, visitadores médicos y los pacientes, en beneficio del sistema de salud y la sociedad peruana. Se sugiere avanzar en la pronta discusión y aprobación de la norma planteada para establecer los criterios éticos de la promoción de productos farmacéuticos y afines, y dar un paso en el desarrollo de las buenas prácticas promocionales ${ }^{(6)}$. De esta forma se evita la omisión de aspectos vinculados a la promoción de comercialización en una industria altamente regulada, especialmente en actividades de desarrollo e investigación, control de calidad y almacenamiento.

Fuente de Financiamiento: Autofinanciado

Conflictos de Intereses: El autor se desempeña como Jefe de Inteligencia Comercial de Medifarma S.A.

\section{REFERENCIAS BIBLIOGRÁFICAS}

1. Chressanthis GA, Khedkar P, Jain N, Poddar P, Seiders MG. Can access limits on sales representatives to physicians affect clinical prescription decisions? A study of recent events with diabetes and lipid drugs: Sales representative access limits to physicians. J Clin Hypertens. 2012;14(7):435-46.

2. Agnetis A, Messina E, Pranzo M. Call planning in European pharmaceutical sales force management. IMA J Manag Math. 2010;21(3):267-80.

3. World Health Organization. Ethical criteria for medicinal drug promotion [Internet]. Geneva, Switzerland: WHO; 1988 [citado el 1 de septiembre de 2017]. Disponible en: http://apps.who.int/iris/bitstream/handle/10665/38125/924154239X_eng.pdf

4. Al-Hamdi A, Hassali M, Ibrahim M. Impact of pharmaceutical promotion on healthcare professional's practices and behaviour: Views from general practitioners, medicine dispensers and medical representatives in Yemen.J Med Mark. 2012;12(4):240-6.

5. Ministerio de Salud. Resolución Ministerial 465-2017 [Internet]. Lima, Perú: MINSA; 2017 [citado el 20 de enero de 2018]. Disponible en: ftp://ftp2.minsa.gob.pe/normaslegales/2017/RM_465-201MINSA.PDF

6. Florián Castro R. ¿Es ética la promoción farmacéutica en el Perú? Gac Sanit. 2016;30(2):159.

Correspondencia: Rafael Florián Castro

Dirección: Av. La Fontana 550. La Molina. Lima. Perú.

Correo electrónico: rafael.florian@usil.pe 